Component-resolved diagnosis in selecting patients for yellowjacket venom immunotherapy

Kukkonen, Anna Kaarina

2018-02

Kukkonen , A K , Pelkonen , A S , Edelman , S M , Kauppi , P M \& Mäkelä , M J 2018 , '

Component-resolved diagnosis in selecting patients for yellowjacket venom immunotherapy '

Annals of Allergy, Asthma, \& Immunology , vol. 120 , no. 2 , pp. 184-189 . https://doi.org/10.1016/j.anai.2017.11.01

http://hdl.handle.net/10138/300967

https://doi.org/10.1016/j.anai.2017.11.012

publishedVersion

Downloaded from Helda, University of Helsinki institutional repository.

This is an electronic reprint of the original article.

This reprint may differ from the original in pagination and typographic detail.

Please cite the original version. 


\title{
Component-resolved diagnosis in selecting patients for yellowjacket venom immunotherapy
}

\author{
Anna Kaarina Kukkonen, MD, PhD; Anna Susanna Pelkonen, MD, PhD; Sanna Marika Edelman, PhD; \\ Paula Maria Kauppi, MD, PhD; Mika Juhani Mäkelä, MD, PhD \\ University of Helsinki and Helsinki University Skin and Allergy Hospital, Helsinki, Finland
}

\section{A R T I C L E I N F O}

\section{Article history:}

Received for publication July 26, 2017.

Received in revised form November 3 , 2017.

Accepted for publication November 14 2017.

\begin{abstract}
A B S T R A C T
Background: Venom immunotherapy is effective in preventing systemic allergic reactions (SARs), but the diagnosis of venom allergy is problematic.

Objective: To compare the performance of component-resolved diagnosis and conventional tests in patients referred for venom immunotherapy.

Methods: We measured serum-specific immunoglobulin E to yellowjacket and honeybee venoms (Ves $\mathrm{v} 1$ and Ves $v 5$ and Api $\mathrm{m} \mathrm{1)}$ ), cross-reactive carbohydrate determinants, serum basal tryptase (ImmunoCAP, ThermoFisher Scientific, Uppsala, Sweden), and skin prick test reactions in 84 patients referred to receive venom immunotherapy. History of SAR and its severity were evaluated.

Results: Of the 78 patients with suspected yellowjacket venom (YJV) allergy, a history of SAR was confirmed in 47 (60\%) and $31(40 \%)$ had a non-SAR reaction. The most accurate tests to confirm venom allergy after a SAR were serum-specific immunoglobulin E to yellowjacket whole-venom extract spiked with Ves $\mathrm{v}$ 5 (area under the curve $0.87,95 \%$ confidence interval $0.77-0.97, P<.001$ ) and Ves v 5 (area under the curve $0.86,95 \%$ confidence interval $0.76-0.96, P<.001$ ). Sensitization to Ves $v 1$ was infrequent and its area under the curve was low $(0.62,95 \%$ confidence interval $0.47-0.76, P=.106)$. Sensitivity of the YJV skin prick test was $86 \%$, but its specificity was low at $54 \%$. Double sensitization to yellowjacket and honeybee occurred frequently in skin prick tests. Of the patients without a SAR, $26 \%$ showed a positive reaction to YJV in any serum test and $46 \%$ showed a positive reaction in skin tests.

Conclusion: Specific immunoglobulin E to the YJV spiked with Ves v 5 confirmed the allergy after a SAR. A history of SAR should be confirmed before testing, because venom sensitization is frequent in other types of reactions.
\end{abstract}

(C) 2017 American College of Allergy, Asthma \& Immunology. Published by Elsevier Inc. All rights reserved.

\section{Introduction}

Hymenoptera venom allergy is a potentially life-threatening condition after an insect sting. Hymenoptera stings cause anaphylaxis more frequently in adults (3\%) than in children $(0.34 \%)$ and account for one fourth of fatalities caused by anaphylaxis. ${ }^{1}$ Systemic mastocytosis increases the risk for severe anaphylaxis to Hymenoptera stings. ${ }^{2-4}$ The risk of a systemic allergic reaction (SAR) to Hymenoptera re-stings is $25 \%$ to $75 \%$ in adults with a previous SAR. The

Reprints: Anna Kaarina Kukkonen, MD, PhD, Skin and Allergy Hospital, Helsinki University Central Hospital, PO Box 160, 00029 HUCH, Helsinki, Finland; E-mail: annakaarina.kukkonen@fimnet.fi.

Disclosures: Authors have nothing to disclose.

Funding Sources: The Helsinki University Research Funds (grant number TYH2016236) and the Finnish Allergy Research Foundation financially supported this study. ThermoFisher Scientific provided ImmunoCAP for serum tryptase analyses. The sponsors had no involvement in the collection, analysis, and interpretation of data; in the writing of the report; or in the decision to submit the article for publication. risk depends on the severity of previous reactions and other known high-risk factors such as age and medication. ${ }^{5}$ Children have lower risk of a SAR at re-stings and their SARs are mild in $60 \% .{ }^{6,7}$

Venom immunotherapy (VIT) with extracted Hymenoptera venoms is effective in preventing a SAR to insect stings: fewer than $3 \%$ of patients treated with vespid VIT have a subsequent SAR. VIT can prevent fatal reactions, and it effectively improves quality of life. ${ }^{8}$ The prerequisite for efficacious VIT is a history of a SAR to an insect sting and immunoglobulin E (IgE) sensitization to the culprit venom.

The assessment of IgE sensitization can be problematic because honeybee (Apis mellifera; Api m) and yellowjacket (Vespula vulgaris; Ves v) venom extracts are complex mixtures of proteins including venom-specific and cross-reactive components. The major allergens are hyaluronidases (Api $\mathrm{m} 2$, Ves v 2), phospholipase A2 (Api m 1), phospholipase A1 (Ves v 1), acidic phosphatase (Api m 3 ), icarapin (Api m 10), and antigen 5 (Ves v 5). ${ }^{6,9}$

Double sensitization to yellowjacket and honeybee venoms as determined by skin prick tests and serum-specific IgE tests is common; up to $50 \%$ of patients show a positive reaction to the 2 
venoms. The major cross-reactive components in honeybee and yellowjacket venoms are hyaluronidases with $50 \%$ sequence identity. Furthermore, cross-reactive carbohydrate determinants (CCDs) cause double-positive test results. ${ }^{6}$ In addition to clinically insignificant positive test results, venom extracts might lack important low-abundance allergens, resulting in false-negative test results.

The distinction between cross-reactivity and true sensitization is important for the choice of VIT, because treating patients who are not sensitized to the allergens in the VIT extract can cause de novo sensitizations, missing protection, and unnecessary costs. ${ }^{9,10}$

The current diagnostic framework in Hymenoptera venom allergy includes skin prick tests, stepwise intradermal testing, and serum-specific IgE to whole-venom extracts and to venomspecific allergen components. ${ }^{11}$ The conventional yellowjacket venom (YJV) extract in ImmunoCAP alone has shown lower sensitivity than the combination of Ves $\mathrm{v} 5$ and Ves $\mathrm{v} 1$. Therefore, it is spiked (sYJV) with Ves $v 5$. When using the sYJV instead of the conventional YJV, the sensitivity increased from $83 \%$ to nearly $97 \%{ }^{12}$ In the US guidelines, venom testing usually requires intradermal testing for optimal sensitivity, and prick tests are optional. ${ }^{5}$ In addition, basophil activation tests and in-house immunoblot tests have been used. Component-resolved diagnostics has improved for the diagnosis of Hymenoptera venom allergy. Measuring specific IgE to allergen components instead of venom extracts helps to distinguish true sensitization from cross-reactivity. We studied the performance of diagnostic tests, including skin prick tests, serum-specific IgE, in-house immunoblot, and serum baseline tryptase, in patients with a strong suspicion of venom allergy referred for VIT.

\section{Methods}

\section{Study Population and Classification of Allergic Symptoms}

The study population of this case-control study consisted of children and adults referred for VIT for suspected Hymenoptera venom allergy. The cases had a SAR to a Hymenoptera sting and the controls had a large local reaction or other type of reaction. The study took place at a single center, a tertiary care hospital. We recruited 30 patients retrospectively from March 2010 through December 2013 and 54 prospectively in 2014. An allergist examined the patients and recorded the history of hymenoptera stings: date, symptoms, signs, emergency visits, and medication. The stinging insect was identified by the patient or by the caregiver. We defined a SAR as a sudden-onset reaction with rapid progression of signs and symptoms involving at least 2 organs including dermatologic (generalized urticaria or erythema, angioedema, or generalized pruritus and skin rash), cardiovascular (hypotension or clinical diagnosis of uncompensated shock), and respiratory (wheeze, stridor, upper airway swelling, or respiratory distress) symptoms. Generalized urticaria without any other symptoms was defined as a SAR in adults. Minor criteria included milder dermatologic, cardiovascular, respiratory, and gastrointestinal signs and symptoms. The definition was based on the Brighton Collaboration Anaphylaxis Working Group suggestion described in detail by Ruggeberg et al. ${ }^{13}$ Severity of the reaction was graded as suggested by Mueller, ${ }^{14}$ referenced in Bilo et al $^{6}$ : grade I was generalized urticaria, itching, malaise, and anxiety; grade II was any grade I reaction plus at least 2 of the following: angioedema, chest constriction, nausea, vomiting, diarrhea, abdominal pain, and dizziness; grade III was any grade II reaction plus at least 2 of the following: dyspnea, wheezing, stridor, dysarthria, hoarseness, weakness, confusion, and feeling of impending disaster; and grade IV was any grade III reaction plus at least 2 of the following: decrease in blood pressure, collapse, loss of consciousness, incontinence, and cyanosis. A large local reaction was defined as a swelling exceeding $10 \mathrm{~cm}$ lasting at least 24 hours.

\section{Allergy Tests}

Skin prick tests were carried out on the patient's inner forearm with a disposable single-use lancet. The yellowjacket and honeybee venoms were tested at 100 and $300 \mu \mathrm{g} / \mathrm{mL}$, respectively (ALKAbello, Hørsholm, Denmark). Histamine chloride $(10 \mathrm{mg} / \mathrm{mL}$ ) was the positive control, and the solvent was the negative control. A reaction wheal of at least $3 \mathrm{~mm}$ was considered positive. We measured serum basal tryptase and specific IgE to SYJV (i3), honeybee venom (i1), CCD (o214), Ves v 1 (i211), Ves v 5 (i209), and Api m 1 (i208; ImmunoCAP, ThermoFisher Scientific, Uppsala, Sweden). We defined sensitization conventionally as a specific IgE level of at least $0.35 \mathrm{kU} /$ L. Since 2012, the conventional YJV ImmunoCAP has been spiked with Ves v 5. ${ }^{12}$ All samples taken before September 2012 were reanalyzed using the sYJV ImmunoCAP. In addition, an in-house Immunospot method ${ }^{15}$ was used to evaluate serum IgE antibodies to YJV and honeybee venom. For the test, $300 \mu \mathrm{g} / \mathrm{mL}$ of Vespula species (ALK-Abelló), 20,000 $\mu \mathrm{g} / \mathrm{mL}$ of Vespula species European mix (Allergon, Ängelholm, Sweden), and $300 \mu \mathrm{g} / \mathrm{mL}$ of A mellifera (ALKAbelló) venoms were used. A nonatopic serum served as a control.

\section{Statistical Methods and Ethics}

The main outcome measure was the accuracy of any diagnostic test in confirming venom allergy in a patient with SAR. We applied receiver-operating characteristics and area under the curve (AUC) to evaluate the performance of the tests in SAR and nonSAR categories. We calculated sensitivity, specificity, and likelihood ratios with $95 \%$ confidence interval $(\mathrm{CI})$. We calculated positive and negative predictive values for specific IgE concentrations. Spearman rank correlation served to correlate severity with the specific IgE concentrations. Mann-Whitney $U$ test was used to compare nonnormally distributed data, and Pearson $\chi^{2}$ or Fisher exact test was used for categorical data. SPSS 21 (SPSS, Inc, Chicago, Illinois) was used for the analyses. The local ethics committee approved the study protocol, according to which the patient (or 1 parent of the child) signed a written informed consent. The study followed the principles of the Declaration of Helsinki.

\section{Results}

Of the 84 patients, 78 were referred for VIT after a yellowjacket sting, 5 after a honeybee sting, and 1 after a sting by an unknown insect. The median age was 44 years (range 1-75); 24 (28\%) were children or adolescents. All children with urticaria (13 of the 23) had other symptoms (dyspnea, vomiting, or angioedema). The performance of the allergy tests was evaluated for yellowjacket allergy in the 78 individuals. Of these patients, 47 (60\%) had experienced a SAR and 31 (40\%) had a non-SAR. The study included only 4 patients with a SAR triggered by honeybee venom. The demographic data and test results of the 78 subjects with a yellowjacket sting are presented in Table 1.

Patients with a SAR to yellowjacket sting were more frequently sensitized to SYJV $(P<.001)$ and Ves v $5(P<.001)$, and they had higher specific $\operatorname{IgE}$ to $\operatorname{SYJV}(P<.001)$ and Ves $v 5(P<.001)$ than those with a non-SAR. The best accuracy was obtained using specific IgE to sYJV (AUC 0.87, 95\% CI 0.77-0.97, $P<.001$ ) and to Ves v 5 (AUC $0.86,95 \% \mathrm{CI} 0.76-0.96, P<.001)$. The AUC of specific IgE to Ves $\mathrm{v} 1$ was lower $(0.62,95 \%$ CI 0.47-0.76, $P=.106$; Fig 1$)$.

Specific IgE to sYJV had the best likelihood ratio (3.2), with $89 \%$ sensitivity and $74 \%$ specificity. Sensitivity of the skin prick test was $86 \%$, but its specificity was lower at $54 \%$. Accordingly, of the patients with a non-SAR to yellowjacket, $46 \%$ were sensitized to skin prick tests and $26 \%$ were sensitized to the serum sYJV test. Sensitivity of the immunoblot was the lowest at $62 \%$. The performances 
Table 1

Baseline Characteristics and Results of Diagnostic Tests of the 78 Patients With Suspected Yellowjacket Venom Allergy

\begin{tabular}{|c|c|c|c|}
\hline & Non-SAR $(n=31)$ & $\operatorname{SAR}(n=47)$ & $P$ value $^{a}$ \\
\hline Age (y), mean (SD) & $39(5-72)$ & $47(1-75)$ & .702 \\
\hline Men, $\mathrm{n}(\%)$ & $7(22)$ & $22(47)$ & .034 \\
\hline Time from sting to blood sampling (mo) & $18(0-175)$ & $3(0-387)$ & .007 \\
\hline Asthma, n (\%) & $12(41)$ & $11(23)$ & .098 \\
\hline Other allergy, $\mathrm{n}(\%)$ & $19 / 31(61)$ & $7(15)$ & .000 \\
\hline \multicolumn{4}{|l|}{ Objective symptoms, n (\%) } \\
\hline Large local reaction & $23(74)$ & $21(45)$ & .010 \\
\hline Hypotension & 0 & $13(28)$ & .001 \\
\hline Loss of consciousness & 0 & $10(23)$ & .011 \\
\hline Vomiting or diarrhea & 0 & $11 / 44(25)$ & .005 \\
\hline Airway obstruction & 0 & $7 / 44(16)$ & .043 \\
\hline Angioedema & 0 & $18(38)$ & .000 \\
\hline Urticaria & 0 & $28(60)$ & .000 \\
\hline Anaphylactic shock & 0 & $9(19)$ & .010 \\
\hline \multicolumn{4}{|l|}{ Subjective symptoms, $\mathrm{n}(\%)$} \\
\hline Tightness of chest & $14 / 25(56)$ & $18 / 44(41)$ & .227 \\
\hline Dizziness & $9 / 25(36)$ & $19 / 44(43)$ & .617 \\
\hline \multicolumn{4}{|l|}{ Treatment of latest reaction, n (\%) } \\
\hline Received glucocorticoids & $11(28)$ & $28(72)$ & .037 \\
\hline Received epinephrine & $4(13)$ & $24(51)$ & .001 \\
\hline Received rescue fluid therapy & 0 & $12(86)$ & .002 \\
\hline Emergency department visit & $14 / 30(48 \%)$ & $37(79)$ & .006 \\
\hline \multicolumn{4}{|l|}{ Serum sIgE } \\
\hline sYJV (kU/L), median (range) & $0.08(0.01-100)$ & $2.76(0.01-100)$ & .000 \\
\hline Honeybee venom (kU/L), median (range) & $0.04(0.01-3.88)$ & $0.05(0.03-36.9)$ & .031 \\
\hline Ves v 1 (kU/L), median (range) & $0.01(0-100)$ & $0.02(0.01-19.7)$ & .108 \\
\hline Ves v $5(\mathrm{kU} / \mathrm{L})$, median (range) & $0.07(0-96.7)$ & $3.48(0.01-100)$ & .000 \\
\hline Api m 1 (kU/L), median (range) & $0.01(0-0.11)$ & $0.01(0-0.48)$ & .850 \\
\hline CCD (kU/L), median (range) & $0.01(0-2.49)$ & $0.01(0-3.09)$ & .92 \\
\hline $\mathrm{sYJV}>0.35 \mathrm{kU} / \mathrm{L}, \mathrm{n}(\%)$ & $8(26)$ & $41 / 46(89)$ & .000 \\
\hline Honeybee venom $>0.35 \mathrm{kU} / \mathrm{L}, \mathrm{n}(\%)$ & $2 / 29(7)$ & $9 / 46(20)$ & .186 \\
\hline Ves v $1>0.35 \mathrm{kU} / \mathrm{L}, \mathrm{n}(\%)$ & $2(7)$ & $5 / 45(11)$ & .694 \\
\hline Ves v 5 > 0.35 kU/L, n (\%) & $10(32)$ & $39 / 46(85)$ & .000 \\
\hline Api m $1>0.35$ kU/L, n (\%) & 0 & $1 / 46(2)$ & 1.00 \\
\hline Double sensitization to sYJV and honeybee whole venom extracts, $\mathrm{n}(\%)$ & $2 / 28(7)$ & $9 / 46(20)$ & .190 \\
\hline $\mathrm{CCD}>0.35 \mathrm{kU} / \mathrm{L}, \mathrm{n}(\%)$ & $2 / 23(9)$ & $4 / 40(10)$ & 1.00 \\
\hline Tryptase $(\mu \mathrm{g} / \mathrm{L})$, median (range) & $4.63(1.10-257)$ & $3.9(1.40-75.8)$ & .191 \\
\hline Tryptase $>13 \mu \mathrm{g} / \mathrm{L}, \mathrm{n}(\%)$ & $3 / 30(10)$ & $5 / 46(11)$ & 1.00 \\
\hline \multicolumn{4}{|l|}{ Skin prick test (titrated) reaction wheal $\geq 3 \mathrm{~mm}, \mathrm{n}(\%)$} \\
\hline YJV & $11 / 24(46)$ & $30 / 35(86)$ & .002 \\
\hline Honeybee venom & $12 / 24(50)$ & $17 / 34(50)$ & 1.00 \\
\hline Double sensitization & $7 / 24(29)$ & $16 / 34(47)$ & .188 \\
\hline \multicolumn{4}{|l|}{ Positive immunoblot test reaction, $\mathrm{n}(\%)$} \\
\hline YJV & $8 / 30(27)$ & $28 / 45(62)$ & .004 \\
\hline Honeybee venom & 0 & $4 / 44(9)$ & .147 \\
\hline Started venom immunotherapy & $5(18)$ & $23(55)$ & .012 \\
\hline
\end{tabular}

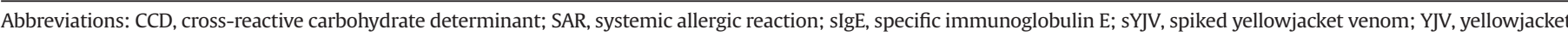
venom.

a $P$ values were determined using Pearson $\chi^{2}$, Fisher exact, or Mann-Whitney $U$ test.

of the diagnostic tests are presented in Table 2. Fig 2 shows the sensitization patterns of the patients with a SAR to yellowjacket sting.

Of the 15 serum samples that were negative to the conventional YJV-specific IgE, 7 (47\%) were positive in the sYJV test spiked with Ves $v 5$.

Double sensitization to yellowjacket and honeybee wholevenom extracts was more common to skin prick tests than to serum tests. Of all patients with suspected YJV or honeybee venom allergy, 24 of 61 (39\%) were doubly sensitized to titrated skin prick tests, and 13 of $82(16 \%)$ were doubly sensitized to serum tests. Sensitization to the CCD did not explain double sensitization in serum tests. Of the double-positive serum tests, 10 (77\%) were positive only to Ves v 5, 3 (23\%) were positive to Ves v 5 and Api m 1, and 8 (62\%) were positive to CCD.

Specific IgE to SYJV correlated strongly to Ves v $5(r=0.98$, $P<.001)$, whereas the correlation to Ves $v 1$ was weaker $(r=0.60$, $P<.001)$. The correlation between Ves $\mathrm{v} 1$ and Ves $\mathrm{v} 5$ was 0.56
$(P<.001)$. The YJV immunoblot correlated weakly to $\operatorname{sYJV}(\mathrm{r}=0.38$ $P<.001)$ and to Ves $v 5(r=0.37, P<.001)$ but did not correlate to Ves $v 1$. Specific IgE to honeybee venom correlated strongly with Api $\mathrm{m} 1(\mathrm{r}=0.98, P<.001)$. Honeybee venom immunoblot showed a moderate correlation to honeybee venom $\operatorname{IgE}(r=0.58, P<.001)$ and to Api $\mathrm{m} 1(\mathrm{r}=0.56, P<.001)$. None of the tests correlated with serum basal tryptase.

Severity of the reaction (grades I-IV) showed a moderate to weak correlation to $\operatorname{SYJV}(\mathrm{r}=0.40, P<.001)$ and to Ves v $5(\mathrm{r}=0.38, P<.001)$. The severity did not correlate to Ves $v 1$ or the size of the skin prick test reaction wheal.

Serum basal tryptase level was increased $(>13 \mu \mathrm{g} / \mathrm{L})$ in $8 \mathrm{pa}-$ tients whose tryptase level ranged from 26 to $257 \mu \mathrm{g} / \mathrm{L}$. Of these patients, 5 (62-75 years old) had mastocytosis and the KIT D816V mutation in bone marrow cells. Of these 5 patients, 4 were diagnosed after the allergic reaction to YJV and serum basal tryptase measurement. 


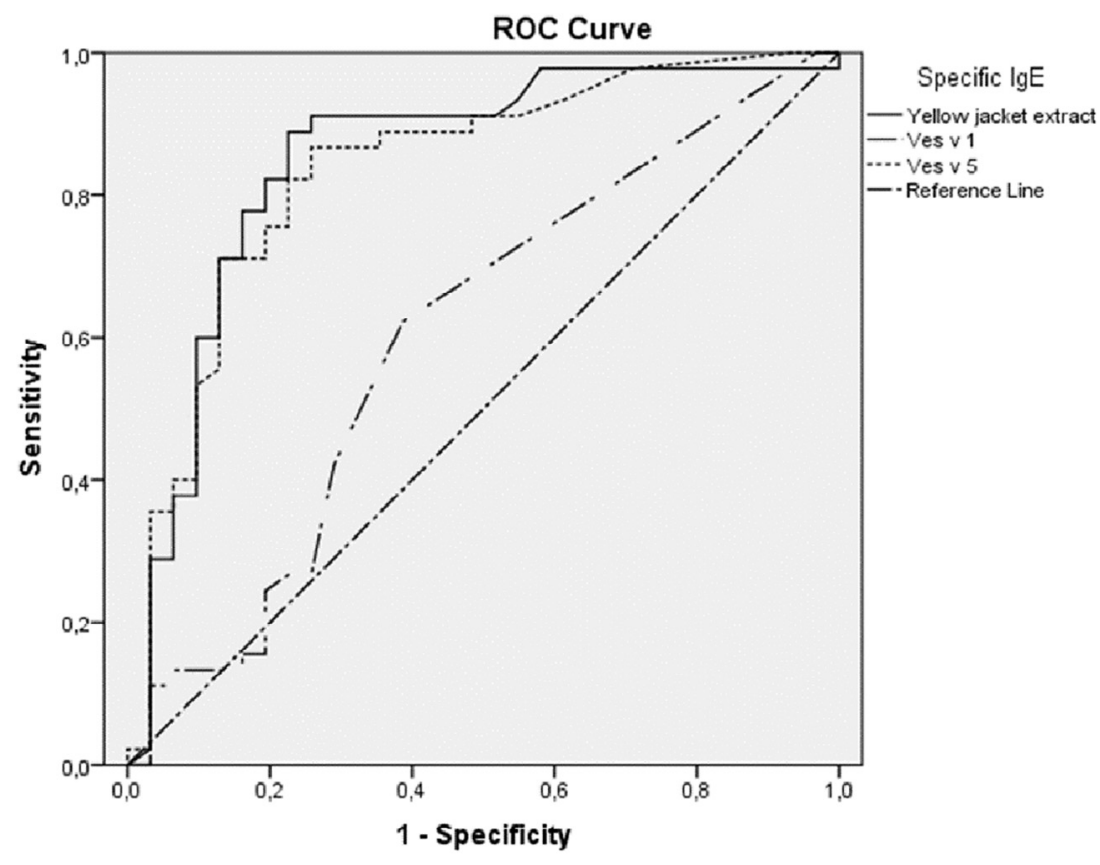

Figure 1. Receiver-operating characteristics (ROC) curve showing the performance of the diagnostic tests for yellowjacket allergy. IgE, immunoglobulin E.

\section{Discussion}

Specific IgE to the yellowjacket whole-venom extract that was spiked with Ves v 5 (sYJV) was superior to other in vitro tests or puncture skin tests in confirming venom sensitization after a SAR. In agreement with previous studies, specific IgE to Ves v 5 performed well, but Ves v 1 added no value. Despite the $86 \%$ sensitivity of skin prick tests, their usefulness was inferior because of the low specificity and large number of double-positive test results to YJV and honeybee venom. The in-house immunoblot test was inferior to serum-specific IgE or skin prick tests.

Measuring specific IgE to the venom allergen components instead of whole-venom extracts has improved the diagnosis of YJV allergy. ${ }^{16}$ Spiking the yellowjacket whole-venom IgE test with Ves v 5 since 2012 has improved its sensitivity. ${ }^{12}$ In our study, $47 \%$ of samples that were negative in the conventional non-sYJV-specific IgE test were positive in the test spiked with Ves $v 5$. Spiking explains the good correlation $(r=0.98)$ between these 2 tests. $^{12}$ Sensitivity of the sYJV was higher (89\%) than that of Ves v 5 alone (85\%), but the combination of these 2 tests yielded $90 \%$ sensitivity. Measuring IgE to the whole-venom extract seems to be useful, because the venom could contain other relevant allergen components. ${ }^{17}$

Unexpectedly, measuring IgE to Ves $\mathrm{v} 1$ added no value in our patients with SAR. Previously, sensitization to Ves v 1 was found in $30 \%$ to $50 \%$ of patients with yellowjacket allergy, ${ }^{14,16,17}$ but monosensitization has been rare.

The weakness of our study is the lack of intradermal testing. Intradermal tests have been highly sensitive and they are recommended as the first-line test in US guidelines. ${ }^{5}$ The sensitivity of skin prick tests is lower than of intradermal tests (86\%), and their usefulness is limited by the frequent double sensitization to yellowjacket and honeybee venoms. Skin prick tests also frequently indicate positivity in patients without a SAR.

Double positivity to the 2 venoms could indicate true double sensitization or could be caused by cross-sensitization. Previously, 50\% to $60 \%$ double positivity has been reported in serum tests. ${ }^{18}$ To our knowledge, little evidence exists on the performance and double positivity of skin prick tests compared with serum-specific IgE tests including recombinant allergen components. Previously, double positivity has been more common in conventional serum IgE tests than

Table 2

Performance of Diagnostic Tests in Patients With a Systemic Allergic Reaction $(n=47)$ and With a Large Local or Other Reaction ( $\mathrm{n}=31)$ to a Yellowjacket Sting

\begin{tabular}{|c|c|c|c|c|c|}
\hline Positive diagnostic test & Sensitivity (\%) & Specificity (\%) & Positive predictive value (\%) & Negative predictive value (\%) & Likelihood ratio \\
\hline \multicolumn{6}{|c|}{$\operatorname{sIgE}>0.35 \mathrm{kU} / \mathrm{L}$ in ImmunoCAP } \\
\hline sYJVa & $89(76-96)$ & $74(55-88)$ & $84(74-90)$ & $82(66-92)$ & $3.4(1.9-6.3)$ \\
\hline Ves v 5 & $85(71-94)$ & $68(48-83)$ & $80(70-87)$ & $75(59-86)$ & $2.6(1.6-4.4)$ \\
\hline Ves v 1 & $11(4-24)$ & $94(78-99)$ & $71(34-92)$ & $42(39-45)$ & $1.7(0.4-8.3)$ \\
\hline sYJV ${ }^{\mathrm{a}}$ or Ves v 5 & $90(78-97)$ & $61(42-78)$ & $79(71-86)$ & $79(61-90)$ & $2.3(1.5-3.7)$ \\
\hline Immunoblot & $62(46-76)$ & $73(54-88)$ & $78(61-90)$ & $56(40-72)$ & $2.3(1.2-4.4)$ \\
\hline \multicolumn{6}{|c|}{ Skin prick test reaction wheal $>3 \mathrm{~mm}$} \\
\hline Titrated $^{\mathrm{b}}$ & $86(70-95)$ & $54(33-74)$ & $73(63-81)$ & $72(51-86)$ & $1.9(1.2-2.9)$ \\
\hline $100 \mu g^{\mathrm{c}}$ & $58(41-75)$ & $62(41-81)$ & $69(55-80)$ & $51(39-64)$ & $1.6(0.9-2.8)$ \\
\hline $300 \mu g^{d}$ & $84(66-94)$ & $59(36-79)$ & $74(63-83)$ & $72(52-86)$ & $2.0(1.2-3.5)$ \\
\hline
\end{tabular}

Abbreviations: sIgE, specific immunoglobulin E; sYJV, spiked yellowjacket venom.

aSpecific IgE to sYJV was measured using an ImmunoCAP test that was spiked with Ves v 5.

bifty-nine test patients.

cFifty-eight test patients.

dFifty-three test patients. 


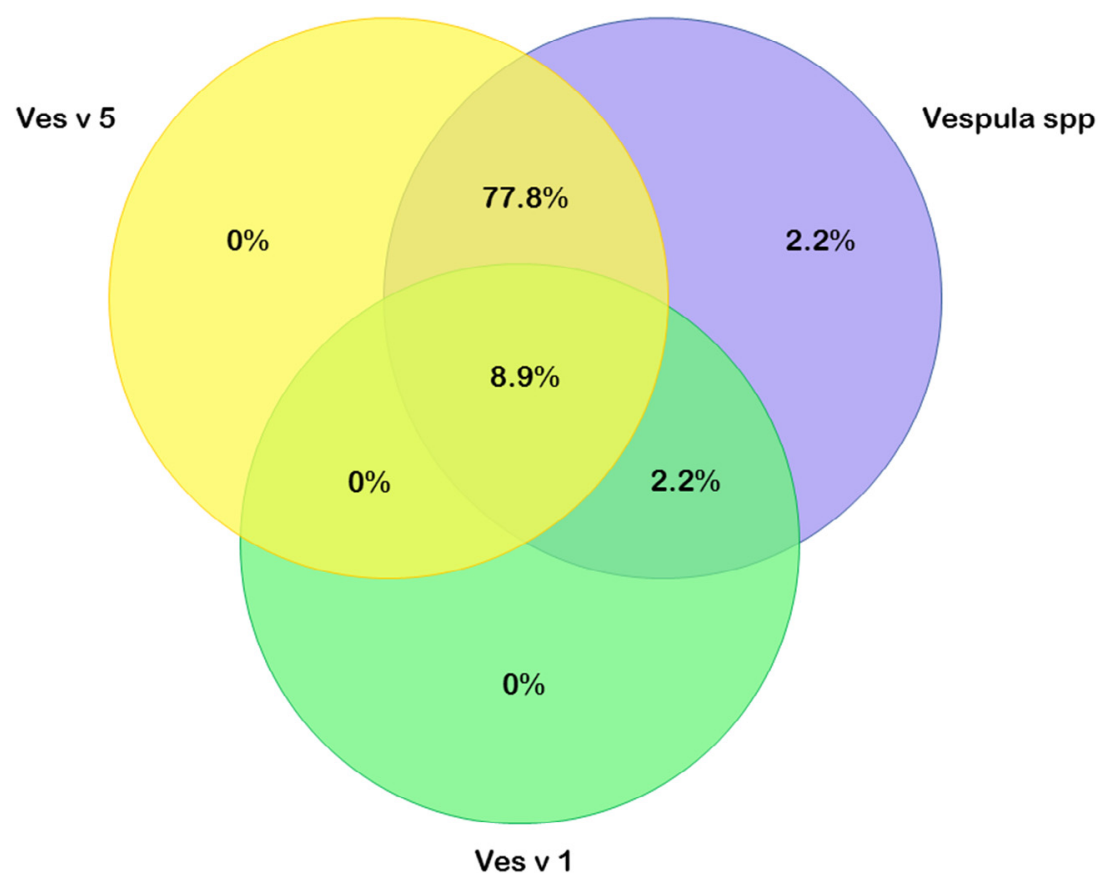

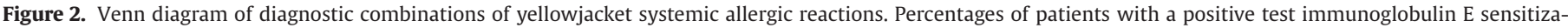
tion to Vespula species extract and Ves v 5 and Ves v 1 allergen components are shown $(n=45)$.

in skin prick tests, ${ }^{19}$ but regional differences can occur in the sensitization profiles of patients with insect venom allergy.$^{20}$ In our study using the sYJV, double positivity to YJV and honeybee venom was much more common in skin prick tests than in serum tests. In agreement with previous studies, double sensitization was mainly independent of CCD sensitization. ${ }^{21}$

In this population with suspected venom allergy, sensitization to sYJV was frequent in patients without a SAR but with a large local reaction, and the specificity of the tests was not optimal. This could be due to the fact that up to $80 \%$ of patients with a large local reaction are sensitized to venom IgE. Up to $46 \%$ of patients without a SAR were sensitized in the titrated skin prick tests, and $26 \%$ were sensitized in conventional serum tests. Although all patients were referred for suspected venom allergy, the thorough history did not always confirm a SAR. Therefore, before screening for venom sensitization, the history of SAR should be confirmed. The prevalence of sensitization to Hymenoptera venoms is estimated to be $10 \%$ to $40 \%$ in the general population. ${ }^{11}$ Sensitized individuals have an increased risk of having a large local reaction to a sting, but the risk for a SAR in sensitized asymptomatic patients is lower than $6 \%{ }^{11}$

The $5 \%$ of our patients who had serum basal tryptase level higher than $20 \mu \mathrm{g} / \mathrm{L}$ were newly diagnosed with systemic mastocytosis and the KIT D816V mutation and fulfilled the World Health Organization minor diagnostic criterion. An anaphylactic reaction to Hymenoptera sting could be an indicator of indolent mastocytosis; therefore, it is useful to measure serum basal tryptase after a SAR to Hymenoptera stings. ${ }^{4,22,23}$

The small number of patients with a SAR to honeybee sting and the lack of commercial tests to Api m 10 and Api m 4 during the study did not allow us to evaluate the performance of the serum tests in honeybee allergy.

The wide interval from the latest sting to blood sampling could affect the results; after an initial decrease (hours), venom-specific IgE increases up to 5 -fold from 1 to 4 weeks after the sting and then gradually decreases. ${ }^{11}$ The results did not change after adjusting for the time interval.
In conclusion, use of a venom-specific IgE measurement should be reserved for patients with a verified SAR who would be considered for VIT. For this indication, in patients with suspected YJV allergy, when intradermal venom skin tests are not performed, the diagnostic tests of choice are serum-specific IgE to SYJV and Ves v 5. Using venom allergen component-specific IgE tests could improve the diagnosis of IgE-mediated YJV allergy, a prerequisite for VIT after a SAR. An in-house immunoblot added no value and should not be used in selecting patients for VIT, because even if the result is positive, the VIT extract might not contain the specific allergens. VIT in patients who are not sensitized to the culprit allergens carries risks and might not be efficient. ${ }^{10}$

\section{Acknowledgments}

Päivi Hanhinen and Pia Sumuvirta are acknowledged for their skillful technical assistance and Soili Mäkinen-Kiljunen is acknowledged for participating in the study design and for fruitful discussions.

\section{References}

[1] Dhami S, Nurmatov U, Varga EM, et al. Allergen immunotherapy for insect venom allergy: protocol for a systematic review. Clin Transl Allergy. 2016;doi:10.1186/ s13601-016-0095-x. [published online ahead of print February 16, 2016].

[2] Guenova E, Volz T, Eichner M, et al. Basal serum tryptase as risk assessment for severe Hymenoptera sting reactions in elderly. Allergy. 2010;65:919-923.

[3] Alvarez-Twose I, Zanotti R, Gonzalez-de-Olano D, et al. Nonaggressive systemic mastocytosis (SM) without skin lesions associated with insect-induced anaphylaxis shows unique features versus other indolent SM. J Allergy Clin Immunol. 2014;133:520-528.

[4] Grabenhenrich LB, Dolle S, Moneret-Vautrin A, et al. Anaphylaxis in children and adolescents: the European Anaphylaxis Registry. J Allergy Clin Immunol. 2016;137:1128-1137, e1.

[5] Golden DB, Demain J, Freeman T, et al. Stinging insect hypersensitivity: a practice parameter update 2016. Ann Allergy Asthma Immunol. 2017;118:28-54.

[6] Bilo BM, Rueff F, Mosbech H, et al. EAACI Interest Group on Insect Venom Hypersensitivity. Diagnosis of Hymenoptera venom allergy. Allergy. 2005;60: 1339-1349.

[7] Lange J, Cichocka-Jarosz E, Marczak H, et al. Natural history of Hymenoptera venom allergy in children not treated with immunotherapy. Ann Allergy Asthma Immunol. 2016;116:225-229. 
[8] Boyle RJ, Elremeli M, Hockenhull J, et al. Venom immunotherapy for preventing allergic reactions to insect stings. Cochrane Database Syst Rev. 2012;(10): CD008838.

[9] Frick M, Fischer J, Helbling A, et al. Predominant Api m 10 sensitization as risk factor for treatment failure in honey bee venom immunotherapy. J Allergy Clin Immunol. 2016;138:1663-1671, e9.

[10] Ollert M, Blank S. Anaphylaxis to insect venom allergens: role of molecular diagnostics. Curr Allergy Asthma Rep. 2015;15:26.

[11] Sturm GJ, Kranzelbinder B, Schuster C, et al. Sensitization to Hymenoptera venoms is common, but systemic sting reactions are rare.J Allergy Clin Immunol. 2014;133:1635-1643, e1.

[12] Vos B, Köhler J, Müller S, et al. Spiking venom with rVes v 5 improves sensitivity of IgE detection in patients with allergy to Vespula venom. J Allergy Clin Immunol. 2013;131:1225-1227, e1.

[13] Ruggeberg JU, Gold MS, Bayas JM, et al. Anaphylaxis: case definition and guidelines for data collection, analysis, and presentation of immunization safety data. Vaccine. 2007;25:5675-5684.

[14] Mueller HL. Diagnosis and treatment of insect sensitivity. J Asthma Res. 1966; 3:331-333.

[15] Leino M, Reijula K, Mäkinen-Kiljunen S, et al. Cladosporium herbarum and Pityrosporum ovale allergen extracts share cross-reacting glycoproteins. Int Arch Allergy Immunol. 2006;140:30-35.

[16] Korosec P, Valenta R, Mittermann I, et al. High sensitivity of CAP-FEIA rVes v 5 and rVes v 1 for diagnosis of Vespula venom allergy. J Allergy Clin Immunol. 2012;129:1406-1408.
[17] Cifuentes L, Vosseler S, Blank S, et al. Identification of Hymenoptera venomallergic patients with negative specific IgE to venom extract by using recombinant allergens. J Allergy Clin Immunol. 2014;133:909-910.

[18] Müller U, Schmid-Grendelmeier P, Hausmann O, et al. IgE to recombinant allergens Api m 1, Ves v 1, and Ves v 5 distinguish double sensitization from crossreaction in venom allergy. Allergy. 2012;67:1069-1073.

[19] Sturm GJ, Bohm E, Trummer M, et al. The CD63 basophil activation test in Hymenoptera venom allergy: a prospective study. Allergy. 2004;59:11101117.

[20] Sturm GJ, Bilo MB, Bonadonna P, et al. Ves v 5 can establish the diagnosis in patients without detectable specific IgE to wasp venom and a possible northsouth difference in Api $\mathrm{m} 1$ sensitization in Europe. J Allergy Clin Immunol. 2012; 130:817, author reply 818-819.

[21] Eberlein B, Krischan L, Darsow U, et al. Double positivity to bee and wasp venom: improved diagnostic procedure by recombinant allergen-based IgE testing and basophil activation test including data about cross-reactive carbohydrate determinants. J Allergy Clin Immunol. 2012;130:155-161.

[22] Ruëff F, Przybilla B, Bilo MB, et al. Predictors of severe systemic anaphylactic reactions in patients with Hymenoptera venom allergy: importance of baseline serum tryptase-a study of the European Academy of Allergology and Clinical Immunology Interest Group on Insect Venom Hypersensitivity. J Allergy Clin Immunol. 2009;124:1047-1054.

[23] Ruëff F, Vos B, Oude Elberink J, et al. Predictors of clinical effectiveness of Hymenoptera venom immunotherapy. Clin Exp Allergy. 2014;44:736746. 